Cahiers d'études africaines

166|2002

Varia

\title{
À propos de l'« africanité » de São Tomé et Príncipe*
}

Elisabetta Maino

\section{OpenEdition}

1 Journals

Édition électronique

URL : https://journals.openedition.org/etudesafricaines/149

DOI : 10.4000/etudesafricaines. 149

ISSN : 1777-5353

Éditeur

Éditions de l'EHESS

Édition imprimée

Date de publication : 1 janvier 2002

Pagination : 385-396

ISBN : 978-2-7132-1429-5

ISSN : 0008-0055

Référence électronique

Elisabetta Maino, "À propos de l'« africanité » de São Tomé et Príncipe* », Cahiers d'études africaines [En ligne], 166 | 2002, mis en ligne le 10 juin 2005, consulté le 14 juin 2021. URL : http://

journals.openedition.org/etudesafricaines/149; DOI : https://doi.org/10.4000/etudesafricaines.149

(C) Cahiers d'Études africaines 


\section{Elisabetta Maino}

\section{À propos de «l'africanité » de São Tomé et Principe*}

Un ouvrage sur São Tomé et Principe est toujours bienvenu, vu la rareté de publication sur ce pays, quelle que soit la langue d'édition. En français, dans le domaine des sciences sociales, nous recensons six thèses dont deux en Droit, une en Lettres, en Théâtre, en Histoire, en Linguistique, et quelques articles ou chapitres à ce sujet. Un dictionnaire portugais-français des particularités, un fablier et un guide touristique nous en donnent un aperçu folklorique usité et soulignent la carence de confrontation disciplinaire sur ce terrain de l'aire lusophone. Les deux essais ci-dessous présentés nous offrent l'opportunité de questionner les nouvelles hypothèses avancées sur ce pays. Ils permettent d'enrichir le débat sur les usages, théoriques et empiriques, des paradigmes épistémologiques par une recherche encore timide sur ce pays.

On appréciera donc l'effort pour traduire vers l'anglais, la thèse en anthropologie culturelle soutenue en janvier 1999 par Gerhard Seibert. L'auteur, qui mène des recherches sur São Tomé depuis 1989, s'inscrit dans le débat sur les changements politiques intervenus dans les pays ayant adopté le modèle de démocratie libérale occidentale et propose l'étude du cas santoméen pour en évaluer l'impact socio-économique et l'interaction avec le modèle préexistant.

Une collecte de matériaux divers (articles de journaux locaux, portugais et autres, rapports de mission d'expertise, tracts, documents d'archives privés et publics), ainsi que les enquêtes de terrain effectuées pendant plusieurs séjours à São Tomé et au Portugal, lui ont fourni un nombre important d'informations. En effet, le principal mérite de ce travail est d'avoir réuni des compilations minutieuses d'événements à partir de différentes sources écrites et orales, ainsi que des annotations personnelles sur les pratiques des acteurs qui interviennent sur la scène économico-politique du pays. On

\footnotetext{
* À propos de Gerhard Seibert, Comrades, Clients and Cousins. Colonialism, Socialism and Democratisation in São Tomé and Príncipe, Leiden University, Research School of Asian, African and Amerindian Studies (CNWS), The Netherlands, 1999 ; Castro Isabel Henriques, Sâo Tomé e Principe. A invençâo de uma sociedade, Lisboa, Vega («col. Documenta Historica »), 2000.
} 
regrettera que telle profusion de renseignements soit livrée à l'état presque de journal de terrain, leur lecture se révélant parfois pénible. Un index analytique des noms propres aurait permis de suivre plus facilement la généalogie et les trajectoires de l'ensemble des personnages de la vie politique santoméenne contemporaine, évitant les répétitions dans les divers chapitres et le double emploi entre le corps du texte et les notes.

Le livre se structure en 9 chapitres, comportant chacun une introduction et une conclusion, chapeautés par une introduction et une conclusion plus générales, le tout accompagné de photographies et d'annexes sur la composition des différents gouvernements du pays depuis son indépendance (1975), ainsi qu'une riche bibliographie. Le découpage des chapitres s'agence tantôt autour d'une logique temporelle (enchaînement des modes d'organisation politique) tantôt sur celle de leurs caractérisations. Une description de l'organisation sociale et de sa modalité de fonctionnement n'apparaît qu'au dernier chapitre (pp. 366-402), tandis qu'un chapitre focalisé sur la situation économique et l'ajustement structurel (chap. 6) s'appesantit sur une série de données financières, livrées en prose. Une présentation plus succincte en aurait permis une lecture plus aérée, d'autant plus qu'il n'y a pas d'analyse de ces éléments, leur fonction se voulant objectivement descriptive.

La partie historique fait largement confiance à quelques textes récents plutôt qu'aux sources originelles. Cela induit des simplifications ou une version univoque des faits, laissant donc la place à de possibles contestations factuelles et interprétatives, attitude que G. Seibert avait adoptée dans une polémique avec Michel Cahen (1993) au sujet des inexactitudes contenues dans un article de ce dernier sur le pays en question.

Les chapitres restants portent sur des phases de transformation politique et économique du pays, passant d'abord par « la construction de l'État socialiste sous le parti unique », pour s'étendre sur la description des processus de constitution des partis dans le système démocratique, ainsi que sur le déroulement des différentes campagnes électorales depuis 1990.

Bien que cela ne soit pas son but explicite, il retrace la structuration de l'élite et des liens qu'elle entretient tant dans l'espace public que privé. S'appuyant sur des notions empruntées à Clapham (1982) (clientélisme), à Bayart (1989) (captation de la rente, stratégie d'extraversion) ou à Olivier de Sardan (1996) (économie morale de la corruption), il examine l'impact du changement institutionnel « de l'autoritarisme socialiste à la démocratie libérale » sur les pratiques et la culture politique de l'élite santoméenne. Cependant, il utilise, en l'ignorant, le concept d'économie morale de la corruption plutôt dans le sens de Médard, qui refuse la neutralité axiologique des analyses positives de Bayart. De plus, ce dernier entend l'extraversion moins comme détournement de capitaux que comme réappropriation d'une idée ou d'une pratique.

La corruption est un élément important dans ce texte, ponctué par de sous-titres tels que : «Compétition pour le pouvoir» (p. 117), «Instabilité politique et invasion » (p. 156), «Les scandales de la corruption » (p. 164), 
«Conflit continuel et rupture finale» (p. 200), «La routine de la corruption » (p. 236), etc. Ce regard moraliste sur la corruption, désormais devenue un sujet de société et de scandale dans les médias de la planète, s'accompagne d'un consensus avec l'analyse qui la considère comme la cause de l'échec des politiques de développement, répandue et reprise par les organismes internationaux de développement, pour qui la lutte contre la corruption est devenue l'enjeu affiché de cette décennie.

L'auteur souligne que l'élite santoméenne, qui avait déjà connu une relative reconnaissance dans l'organisation sociale dès le $\mathrm{XVI}^{\mathrm{e}}$ siècle, se trouva déplacée dans les bas rangs de la bureaucratie coloniale et cultiva donc des aspirations nationalistes, renforçant l'identité forro ${ }^{1}$ qu'il différencie de celle des travailleurs des plantations (recrutés de force, principalement dans les autres colonies portugaises, ils remplacèrent les esclaves). Ces derniers ne semblent pas avoir d'identité, et encore moins d'aspirations; au plus, ils sont définis comme Capverdiens; le jus solis n'ayant pas de place dans la vision ethniciste. On pourrait donc supposer qu'ils échappent ainsi à la taxinomie qui fait de tout Forro un oisif. Il n'en est rien ; en effet, reprenant à son compte l'image du natif voleur et indolent que la littérature coloniale avait forgée (pp. 32, 45, 131, 138, 140, etc.), dans le constat que l'aversion au travail, spécialement agricole, est une constante, il repère la cause de la baisse de production agricole et des mauvais résultats économiques de la période postcoloniale dans « le non-remplacement de la discipline du travail forcé par l'éthique du travail productif » (pp. 139, 151). Il caractérise cette période par les carences en ressources humaines disposant de la formation et du savoir-faire adéquats (reprenant ainsi les rapports d'expertise préalables à l'assistance technique des bailleurs de fonds), auxquelles il ajoute l'autoritarisme, la bureaucratisation et le clientélisme du Parti unique. Cet amalgame d'éléments requiert un débroussaillage: les Forros, indolents par essence, ne peuvent être tenus pour responsables de la baisse d'une production qu'ils n'ont jamais favorisée, tout comme les travailleurs (ex-forcés) ne sauraient être accusés de manquer d'éthique du travail. Or, de quoi parle-t-on ? De la bureaucratisation de l'élite ? De l'incompétence des travailleurs agricoles ? D'un Parti unique autoritaire qui ne sait pas imposer la discipline ?

Selon G. Seibert « l'adoption du modèle démocratique de l'économie de marché et du multipartisme a révélé que les obstacles au développement du pays sont à rechercher dans les facteurs internes » (p. 290), c'est-à-dire dans les formes d'organisation sociale propres à cette société, tout compte fait très africaine par ses mœurs relâchées (chap. 9).

Sa thèse est qu'il y a une continuité dans la culture politique du pays, attendu que le système autochtone a largement influencé le modèle de multipartisme introduit de l'extérieur. L'échec de la politique économique de

1. Avant l'abolition de l'esclavage en 1875, le terme forro désignait les affranchis et, ensuite, les natifs ayant fait souche. 
développement est expliqué, mis à part la litanie usuelle des carences structurelles, par la lourdeur bureaucratique interne et l'incapacité d'organisation à tous les niveaux et dans tous les secteurs de la société. Le succès de la démocratie libérale serait dû à l'habileté des partis en compétition à maintenir la lutte pour le pouvoir politique dans un cadre institutionnel (p. 242). Néanmoins, le non-respect des normes serait profondément enraciné dans la culture locale (p. 244). Les aides auraient profité aux leaders politiques, captateurs de rente pour leur consommation personnelle ou pour une redistribution clientéliste intra-forro, plutôt que pour l'investissement productif du pays. L'élite aurait réussi à se reconvertir et à adapter ses stratégies de lutte pour le pouvoir au multipartisme, notamment en s'impliquant elle-même dans des rapports de patronage à l'échelle internationale, pour capter des fonds externes et, au niveau interne, en renforçant ses capacités de captation de votes. Pour ce faire, le recours aux réseaux de parenté et d'allégeance clientéliste serait la norme, ainsi que l'utilisation de la rumeur pour dénigrer les rivaux dans les campagnes électorales. L'auteur présume que si la rumeur ne mène pas à des conflits ouverts, c'est parce que les gens évitent le conflit pour faciliter la coexistence dans un espace, tout compte fait, limité. À quoi bon, donc, dédier un chapitre à la rumeur si, en définitive, elle n'a aucune répercussion dans la vie politique et sociale?

G. Seibert constate que l'argent est une composante importante dans la compétition politique et que l'activité militante ne répond pas à des convictions, mais qu'elle est souvent pratiquée comme emploi rémunéré (p. 325). Néanmoins, il admet que la distribution de cadeaux n'assure pas les votes, comme le montrent les résultats des élections, dont « le score des candidats répond à leur réputation locale », ce qui invalide son analyse sur le clientélisme à fin électoraliste. Lui-même écrit que « les résultats du suffrage montrent que la société n'est pas soumise à la pression sociale ou d'un groupe dans les élections, et les individus bénéficient d'une considérable liberté individuelle dans le choix politique » (p. 326). Son analyse serait donc prisonnière de ses propres convictions sur l'importance du clientélisme et de la corruption, au point de négliger ses propres constatations. Il rajoute que ce cas confirme les six thèses de Olivier de Sardan, selon lesquelles « il n'y a pas de corrélation entre les degrés de corruption et les modèles de système politique » (p.411). La démocratie aurait favorisé la transparence et la dénonciation de pratiques de corruption qui se développent grâce à la persistance de logiques culturelles clientélistes et au pragmatisme normatif. Or, il est évident que dans un micro-pays (130000 hab.) l'élite socioéconomico-politique ne fait qu'un et que les réseaux se chevauchent, que les intérêts et les allégeances se côtoient. On remarquera son insistance sur le particularisme de la culture forro, sans pour autant nous dire en quoi elle se différencie de celle des non-forros, population décrite en termes misérabilistes et ghettoïsée dans les plantations.

En raison de la faible codification sociale des normes concernant l'institution familiale, il est contradictoire d'indiquer que «les liens de parenté 
structurent des rapports de loyauté sur cette base» (p.414), comme le montre le fait «qu'on puisse appartenir à une même famille sans pour autant être membre du même parti, ni défendre les mêmes intérêts » (p. 403). Ainsi, un service rendu (facilitation d'emploi, de service, d'argent) ne se traduit pas par une allégeance sine die, puisqu'on garde toujours la possibilité du libre choix. La non-exclusivité des liens permet, en effet, d'agencer des alliances, selon les situations et les contextes. Si les électeurs demandent des cadeaux pour faire campagne pour un candidat donné, sans forcément adhérer aux idées affichées par son parti, ne serait-ce pas plutôt que, puisqu'il n'y a «pas de différences essentielles dans les programmes proposés » (pp. 324-325), les électeurs, tout comme G. Seibert, ne sont pas dupes ? L'illettrisme n'est pas synonyme de stupidité, tout comme le multipartisme n'est pas synonyme de démocratie.

Finalement, avec un optimisme inespéré, il conclut que le caractère pacifique des Santoméens, leur faible niveau de pluralisme culturel (dans le sens d'hétérogénéité ou d'homogénéité ?), autant que leur capacité d'adaptation à des nouveaux modèles politiques, sont des atouts pour la consolidation de la démocratie libérale, si toutefois celle-ci s'accompagne d'une croissance économique et du renforcement du rôle de la loi, afin de limiter la portée du parrainage dans les rapports sociaux.

Isabel Castro Henriques, chargée de la chaire d'Histoire de l'Afrique à la faculté de Lettres de l'Université de Lisbonne, reprend un thème déjà traité dans ses publications antérieures, qui s'inscrit dans la relecture critique du rôle du Portugal dans l'émergence de l'économie-monde. Le titre et l'édition sur papier patiné, enrichie d'une abondante iconographie (128 illustrations dans 151 pages !) portant aussi bien sur des époques postérieures à celle traitée que sur d'autres situations, peuvent donner une impression de célébration. Elle nous précise que la vocation de ces images «n'est pas de falsifier l'histoire mais d'éclaircir des situations historiques par leur analogie avec des réalités similaires » (p. 12), similarité qui pourrait être perçue comme contradictoire avec l'idée avancée de «la singularité de ce projet portugais d'outre-mer».

Son propos est de montrer comment la création de la société santoméenne aux $\mathrm{XV}^{\mathrm{e}}$ et $\mathrm{XVI}^{\mathrm{e}}$ siècles est le résultat de la convergence de deux opérations: la modification de l'écosystème et l'invention de nouvelles structures sociales, dans lesquelles «l'initiative portugaise a été le moteur, mais ce sont les Africains, via les Métis, qui en ont marqué le rythme ». On remarquera que le terme Africain remplace celui de Noir, terme récurrent dans les sources documentaires référencées, tandis que celui de l'Européen est utilisé comme synonyme de Portugais, terminologie habituelle dans la littérature savante portugaise. Ce choix nous indique la volonté de dépasser une relecture historique raciale à l'aune d'une vision moderniste qui, prétendant s'affranchir d'un passé colonial, affirme une appartenance à un patrimoine géographico-culturel originel qui en devient sa justification, retrouvant une continuité avec un passé de valeurs et de pratiques particulières. 
L'essai s'articule en 5 chapitres. Le premier met l'accent sur les conditions d'occupation dans le cadre des découvertes. São Tomé et Principe (1470-1471) ne sont pas les seules îles inhabitées de l'Atlantique que les Portugais «trouvèrent », mais elles retiennent plus particulièrement l'attention de l'auteur du fait que, avec celles du Cap-Vert, «elles furent les seules à être retenues pour une expérience novatrice en matière de peuplement comportant des Africains » (p. 24), puisque «Madère, Porto Santo et les Açores furent peuplées presque exclusivement par des Blancs européens ou d'Afrique du Nord » (p. 24 note 11). Or, cette comparaison postule qu'il y eut une nette volonté politique de différencier les modalités de peuplement, lorsque c'est plutôt la combinaison de plusieurs facteurs non maîtrisés qui en a orienté l'évolution.

Le deuxième chapitre reprend la question du peuplement et en examine les composantes humaines dans une mise à plat de la structuration sociale, qui met l'accent plutôt sur la diversification des groupes sociaux que sur l'imbrication des conflits engendrés par les désirs de mobilité intergroupes. Les privilèges offerts par la Couronne portugaise n'attirèrent pas un nombre suffisant de colons blancs. Ainsi, en 1506, la plupart des 200 Blancs installés sont des déportés portugais condamnés à la relégation en alternative à la prison ou à la mort, qui se voient attribuer, en plus des conditions pour s'enrichir, des esclaves noires afin d'assurer le peuplement. À ce groupe s'ajoutent les fonctionnaires préposés aux tâches administratives et militaires, dont les demandes de retour à la métropole témoignent du caractère contraignant de ce séjour, et enfin, des Castillans, des Français ou des Génois, dont la présence en 1540 ne nous semble pas suffisante pour démontrer le caractère persévérant de «la participation d'Européens » (p. 35). Les Africains sont majoritaires, généralement esclaves, mais aussi libres, soit par naissance soit par affranchissement. Généralisant le cas «d'hommes riches avec qui les Portugais établissaient des relations sur le littoral africain » venus s'installer volontairement, et celui de Noirs libres économiquement insérés, l'auteur en déduit la conjonction d'intérêts avec les Portugais « qui s'appuyèrent sur les connaissances des hiérarchies sociales des responsables africains pour coloniser l'île» (p. 44). Cependant, si on répertorie des Métis (la plupart) et quelques Noirs parmi la couche des commerçants ou des planteurs, la reconnaissance de leur position sociale n'était pas acquise d'emblée, comme l'indiquent les conflits à ce sujet. L'exploitation outrancière poussait à la révolte les esclaves, dont les refuges dans la forêt furent appelés mocambo. Dans les rapts de femmes effectués par ces esclaves fugitifs, l'auteur lit, outre une pratique courante en Afrique, une volonté d'assurer la pérennité du mocambo et d'africaniser l'île (p. 57 note 47). I. C. Henriques rejette la légende qui désigne les Angolares comme des Noirs rescapés d'un naufrage ayant mené des attaques répétées contre les plantations, puisque « leur attribuer la responsabilité de la résistance aux Européens reviendrait à réfuter la thèse de l'île inhabitée et inconnue par 
les Africains ». Elle ajoute que cette hypothèse, reprise aussi bien par l'idéologie coloniale (quel intérêt de fomenter l'idée d'une possible présence humaine antérieure à celle portugaise ?) que par «les histoires nationalistes africaines » (dont l'intérêt de reprendre un récit qui légitime leur passé d'autonomie et de résistance est beaucoup plus compréhensible), est invalidée par «le caractère dépassé de la question du premier arrivé », l'antériorité de la présence des Angolares étant un faux problème puisque "ce sont les Africains avec les Européens et les Métis qui ont consolidé la socialisation » (pp. 59-60).

Le troisième chapitre traite de la socialisation de l'espace à travers la culture de la canne à sucre, qui engendra le réaménagement de l'écosystème et le recours au travail des esclaves. Le Portugal, «à qui l'Europe a délégué la tâche des découvertes du monde et d'en diriger l'organisation et l'exploitation économique », choisit São Tomé comme centre d'expérimentation où sont mises à contribution les connaissances des uns et des autres (pp. 6364). Ainsi, le Portugal se charge de la maîtrise technique de la production et du transport du sucre, tandis que l'Afrique apporte la main-d'œuvre nécessaire, Le recours à l'esclavage allait de soit, il s'inscrivait dans le prolongement « du commerce d'esclaves noirs que les Arabes pratiquaient déjà dans la Méditerranée » (p. 67). Dans un souci de rentabilité, l'organisation du travail sut prendre en compte « les particularismes culturels » (p. 84) des esclaves, d'une part en préférant l'achat par couple, ce qui garantissait la stabilité psychologique et la reproduction de la main-d'œuvre et, d'autre part, leur permettant de consacrer un jour par semaine à l'agriculture de subsistance, ce qui non seulement était «plus rentable puisqu'il permettait de bénéficier d'une force de travail nombreuse et auto-alimentée », mais, de plus, introduisait «un régime de travail originaire de l'Afrique » aussi bien en matière de produits et de techniques utilisés (p. 81) que dans une relative continuité avec les formes de division sexuelle du travail, dans laquelle les femmes se chargent de la plupart des tâches agricoles, comme le suggère la note 45 et les illustrations $n^{\circ} 75$ et 76 (pp. 84-85). L'auteur réfute l'interprétation de Tenreiro (1961), qui qualifie ce régime de servage moyenâgeux. Cependant, elle oublie que l'exploitation des compétences des esclaves n'était ni spécifique à la canne à sucre ni à ce territoire (Viana Filho 1988 ; Heers 1981 : pp. 135-164 ; Folhlen 1998 : pp. 153-189), mais une forme d'organisation du travail esclave conforme à des capitaux réduits ou à la disponibilité locale d'autres facteurs, répandue aussi dans les Amériques (Furtado 1970 : chap. VI ; Freitas 1983).

Le quatrième chapitre sur « l'africanisation » montre que la polyvalence des fonctions assignées à São Tomé - centre d'introduction de plantes et techniques nouvelles; point d'appui et approvisionnement des navires ; stockage des esclaves destinés au continent et au Nouveau Monde — n'aurait pas été possible sans l'intervention, volontaire ou forcée, des Africains. Leur collaboration fut mise à profit non seulement dans la traite, mais encore 
dans la production vivrière destinée au marché interne et externe. I. C. Henriques soutient que la création d'une population métisse est un autre point qui montre 1'activisme des Africains. Elle oppose cet argument à celui qui assigne «tout le dynamisme aux Portugais et transforme les femmes noires en simples objets sexuels » (p. 103 note 14). «Si l'idée initiale de créer des Métis était bien portugaise [...] les considérant comme indispensables et adaptés au contexte écologique local » (p. 104), les Africains ne se limitaient pas à générer de «faux Blancs mais élevèrent des Métis culturellement marqués par la famille africaine, qui assurait leur socialisation» (p. 105). S'appuyant sur la phrase d'un manuscrit de 1540 qui indique plutôt l'acculturation des Noirs aisés : «Les commerçants blancs n'hésitent pas à prendre pour femme les filles que les Noirs riches élèvent selon nos coutumes » (1540) et sur la description du respect du rituel (africain ?) de la queue par les prétendants blancs des jeunes filles noires, à Luanda (1792), l'auteur conclut que les Métis nés de ces unions ne sont pas le fruit de relations imposées, mais de consentement mutuel. «C'est le mariage, valorisé tant par les Portugais que par les Africains, qui institutionnalise les alliances », passant ainsi sous silence le fait que le mariage ne concernait qu'une frange infime de la société, négligeant le fait que les pétitions d'affranchissement ou de reconnaissance des droits d'héritage nous parlent d'enfants illégitimes et déniant la condition historique de subordination des femmes, quelle que soit la structure familiale ou sociétale. Des photographies de 1895 ( ${ }^{\circ} 100$ 102) montrent des Noirs aisés : or, au-delà du fait de l'extrapolation de quelques siècles, elles dévoilent plutôt leur «européanisation », tant dans l'habillement que dans les fonds scénographiques, comme l'indiquent aussi les légendes des images.

La visée de l'ouvrage est de montrer que les Africains ont joué sur les deux tableaux : d'une part les Métis ont poursuivi des stratégies d'assimilation aux Européens en se faisant reconnaître comme les authentiques « enfants de la terre» mais, enracinés dans les valeurs maternelles, ont contribué à la création d'un système culturel à prépondérance africaine. En parallèle, les esclaves fugitifs ont progressivement occupé et contrôlé les espaces non investis par les Portugais, afin de «préserver les valeurs africaines » (p. 114). Il faudrait souligner que :

- le pourcentage de Métis (1\%) a été aussi insignifiant numériquement que celui des Blancs $(0,3 \%)$;

- l'édit de peuplement ne stimulait pas le mariage interracial mais ordonnait de donner une esclave aux premiers colons portugais;

— ce «projet» de créer des Métis n'en mesurait pas les conséquences, comme en témoignent les différends à leur sujets ;

- mis à part les chartes royales accordant l'affranchissement aux descendants des premiers colons (1515 et 1517), les suppliques d'émancipation eurent un caractère ponctuel et individuel ;

- les acquiescements étaient inférieurs aux pétitions de reconnaissance de filiation; 
- la persistance de la catégorie « Métis » dans les recensements des années 1960 montre qu'il n’y a jamais eu d'intégration dans les groupes des Blancs ou des Noirs ;

- si le principe de mixité avait été admis, on pourrait trouver des unions entre femmes blanches et hommes noirs, libres ou non, ce qui n'était évidemment pas le cas.

Le cinquième chapitre conclue que, face à l'échec du projet de colonie de peuplement, car les Métis n'ont pas pris «la relève pour perpétuer les valeurs portugaises et sont devenus des ennemis » (pp. 122-123), et face aux difficultés de la Couronne portugaise, les planteurs auraient jeté l'éponge pour aller s'installer au Brésil, laissant à ce pays désormais africanisé, proie des convoitises corsaires, le rôle d'escale des bateaux négriers. Le retour des caravelles aura lieu au XIX ${ }^{\mathrm{e}}$ siècle, lorsque du Brésil on importa le café et le cacao et, puisque «les plantes sont porteuses d'un système », une deuxième vague d'organisation de la monoculture pour l'exportation adopta non plus la violence de l'esclavage, mais le recours aux «faux travailleurs libres ». Avec le constat du triste fait que «l'inévitable violence de la domination coloniale, dont ces plantes sont les agents, trouve aujourd'hui son leg dans la violence du marché », on replonge dans l'inexorabilité du déterminisme structurel.

Le renvoi au caractère bioculturel tranche donc le débat cher à l'historiographie portugaise, qui défendait le caractère national-patriotique des provinces d'outre-mer. L'auteur constate que les Portugais se sont aperçus de leur incapacité à maîtriser commercialement et socialement ce territoire, tandis que les Africains ont réussi à intégrer des propositions européennes, et le résultat est une «nouvelle société caractérisée par le métissage des hommes, des systèmes économiques, des connaissances, des techniques et des plantes » (p. 125). Les Portugais, médiateurs de l'Europe, partagent avec des chefs noirs le projet d'inventer un nouvel Homme pour le monde émergent. Ce climat de coopération économique et d'échanges culturels prendra une coloration aux tonalités africaines. Lamentablement, il était gâché par la mauvaise coutume de l'époque qui fut l'esclavage, dont le capitalisme naissant est responsable.

Dans le louable effort de revaloriser l'apport de l'Afrique dans la scène de l'Histoire, il y a un dérapage d'interprétation culturaliste du biologique. Le métissage est certainement une réalité, mais en faire l'apologie, sous prétexte de collaboration d'une frange sociale, n'efface-t-il pas son caractère de contrainte ? Dans quelle mesure peut-on parler de participation active des esclaves au processus de socialisation?

L'absence des données quantitatives et, d'une certaine façon, qualitatives devrait pousser les chercheurs à être attentifs dans la formulation des hypothèses explicatives : en effet, les sources principales des exégèses sont deux relations de marins : le manuscrit de Valentin Fernandez de 1506 et celui connu comme le Pilote portugais anonyme daté de (1540). Ces deux 
textes, avec quelques pièces de sources administratives ou religieuses constituent la documentation sur laquelle s'appuie tout argument ou démonstration concernant le $\mathrm{XVI}^{\mathrm{e}}$ siècle. Ainsi, toute généralisation ou décontextualisation des informations par rapport à des faits spécifiques engendre une surinterprétation aboutissant à des contresens, qui invalident la portée scientifique des arguments avancés.

La pléthore de qualificatifs comme «inédite, nouvelle, originale, particulière » met l'accent sur la volonté explicite de créer une réalité aux caractéristiques singulières. Le mélange de l'aspect écologique et humain en donne une vision botanique dans laquelle on perd de vue l'organisation politique, qui est, bien plus que la flore, un facteur déterminant de la construction sociale. La circulation d'une technique culturale ou d'une pratique alimentaire ne comporte pas forcément des phénomènes d'acculturation ou d'inculturation. Qu'est-ce que les pommes de terre ont véhiculé en Europe de la culture andine? Peut-on prétendre que les Africains sont plus asiatiques pour avoir inclus dans leur alimentation le riz?

São Tomé n'aurait-t-elle pas été "africanisée » parce que numériquement les Noirs ont toujours été majoritaires et que dans nos représentations le Noir est africain? Les Antilles sont-elles africanisées vu l'importance de la population noire? Pourquoi asseoir la sédimentation de São Tomé aux $\mathrm{XV}^{\mathrm{e}}$ et $\mathrm{XVI}^{\mathrm{e}}$ siècles, alors que l'essor de peuplement n'a eu lieu qu'à la fin $\mathrm{du} \mathrm{XIX}^{\mathrm{e}}$ ? Pour fonder historiquement les marqueurs culturels dans son passé, au risque d'ainsi conforter d'éventuelles généalogies d'autocthonie ?

La formation d'une société est le résultat d'un projet mais aussi de l'aléatoire, de l'imprévisible, le produit de la combinaison des dynamismes internes et externes avec lesquels il faut sans cesse réajuster non seulement les tactiques mais encore les objectifs. Dans ce processus rhizomatique de métissage, il importe moins de savoir qui a apporté quoi que d'analyser les mécanismes d'appropriation et de réinterprétation des logiques pour appréhender le sens spécifique qu'une société se donne. En définitive, la question de savoir sur quelle base ethnique-culturelle une société est construite dans le passé ne légitime pas la présence de qui y vit maintenant et comment on s'y organise au présent. La légitimité de la souveraineté santoméenne ne réside pas tant dans le fait que le pays ait été africanisé depuis le $\mathrm{XVI}^{\mathrm{e}}$ ou le $\mathrm{Xx}^{\mathrm{e}}$ siècle, mais dans le fait que ses habitants actuels s'identifient dans une communauté de citoyens qui s'autogouvernent, peu importe leur degré d'africanité.

Doctorante EHESS, CEAF. 


\section{BIBLIOGRAPHIE}

BAYART, J.-F.

1989 L'État en Afrique : la politique du ventre, Paris, Fayard.

CAHEN, M.

1991 «Arquipélagos da alternância: a Vitoria da oposição nas ilhas de Cabo Verde e de São Tomé e Príncipe », Revista internacional de estúdios africanos, 14-15, Lisboa, IICT : 113-154.

1993 «Correspondencia», Revista internacional de estúdios africanos, 16-17: 353-365.

Clapham, C.

1982 «Clientelism and the State», in C. Clapham, ed., Private Patronage and Public Power: Political Clientelism in the Modern State, London, Frances Pinter.

FERNANDEZ, V.

1506-1510 O manuscrito..., annoté par L. Baiao, Lisboa, Academia Portuguesa de Historia, 1940 (trad. in Th. Monod, A. Teixeira da Mota, R. Mauny (dir.), Description de la Côte ocidentale d'Afrique, Bissau, CEGP, 1951).

Folhlen, C.

1998 Histoire de l'esclavage aux États-Unis, Paris, Perrin.

FREITAS, D.

1983 Escravos e senhores de escravos, Porto Alegre, Mercado Aberto.

Furtado, C.

1970 [1959] La formatione economica del Brasile, Torino, Einaudi.

HEERS, J.

1981 Esclaves et domestiques au Moyen Âge dans le monde méditerranéen, Paris, Fayard.

HenRiques, C. I.

2000 Sâo Tomé e Principe. A invençâo de uma sociedade, Lisboa, Vega («col. Documenta Historica »).

MÉdARD, J.-F.

2000 «Clientélisme politique et corruption», Revue Tiers-Monde, 161:75-88.

s. d. Navegação de Lisboa à ilha de São Thomé escrita por um piloto português (século XVI), annoté par A. Reis Machado, Lisboa, Portugalia, (s.d.).

Olivier DE SARDAN, J.-P.

1996 «L'économie morale de la corruption en Afrique », Politique africaine, 63 : 97-116. 
SEIBERT, G.

1999 Comrades, Clients and Cousins. Colonialism, Socialism and Democratisation in São Tomé and Príncipe, Leiden, Leiden University, Research School of Asian, African and Amerindian Studies (CNWS).

TEnREIRO, F.

1961 A ilha de São Tomé, Lisboa, Junta de Investigações do Ultramar.

ViAnA Filho, L.

1988 [1944] O negro na Baia, Rio de Janeiro, Nova Fronteira.

\section{RÉSUMÉ}

La lecture critique de deux ouvrages sur São Tome et Príncipe, le plus petit des cinq pays africains de langue officielle portugaise (palop), nous permet de questionner la pertinence de la notion d'africanité.

\section{ABSTRACT}

On the "Africanicity" of São Tomé and Príncipe. - A critical reading of two books on São Tome et Príncipe, the smallest of the five African countries that have chosen Portuguese as official language, serves as a basis for questioning the relevance of the notion of "Africanicity".

Mots-clés/Keywords : Afrique lusophone, São Tomé et Príncipe, africanité, colonialisme, corruption, démocratisation, esclavage, métissage, multipartisme, plantation/ Africa, São Tomé et Príncipe, africanicity, colonialism, corruption, democratization, slavery, métissage, multiparty politics, plantation. 